\title{
Publisher Correction: Intrinsic plasticity of silicon nanowire neurotransistors for dynamic memory and learning functions
}

Eunhye Baek (D), Nikhil Ranjan Das (1), Carlo Vittorio Cannistraci, Taiuk Rim, Gilbert Santiago Cañón Bermúdez, Khrystyna Nych, Hyeonsu Cho (1D, Kihyun Kim, Chang-Ki Baek (1), Denys Makarov @ , Ronald Tetzlaff, Leon Chua, Larysa Baraban (D) and Gianaurelio Cuniberti id

Correction to: Nature Electronics https://doi.org/10.1038/s41928-020-0412-1, published online 25 May 2020.

In the version of this Article originally published, in equation (9), the second $\Delta I_{\mathrm{d} 2}$ was incorrect; it should have been $\Delta I_{\mathrm{d} 1}$. All versions of the Article have been amended.

Published online: 16 June 2020

https://doi.org/10.1038/s41928-020-0442-8

(C) The Author(s), under exclusive licence to Springer Nature Limited 2020 